\title{
LATTICE METHODS FOR QUANTUM CHROMODYNAMICS
}


This page is intentionally left blank 


\section{LATTICE METHODS FOR QUANTUM CHROMODYNAMICS}

\section{Thomas DeGrand}

University of Colorado, USA

Carleton DeTar

University of Utah, USA 
Published by

World Scientific Publishing Co. Pte. Ltd.

5 Toh Tuck Link, Singapore 596224

USA office: 27 Warren Street, Suite 401-402, Hackensack, NJ 07601

UK office: 57 Shelton Street, Covent Garden, London WC2H 9HE

\section{British Library Cataloguing-in-Publication Data}

A catalogue record for this book is available from the British Library.

\section{LATTICE METHODS FOR QUANTUM CHROMODYNAMICS}

Copyright $\odot 2006$ by World Scientific Publishing Co. Pte. Ltd.

All rights reserved. This book, or parts thereof, may not be reproduced in any form or by any means, electronic or mechanical, including photocopying, recording or any information storage and retrieval system now known or to be invented, without written permission from the Publisher.

For photocopying of material in this volume, please pay a copying fee through the Copyright Clearance Center, Inc., 222 Rosewood Drive, Danvers, MA 01923, USA. In this case permission to photocopy is not required from the publisher.

ISBN $981-256-727-5$

Printed in Singapore by B \& JO Enterprise 
to Laurel and Mary Ann 
This page is intentionally left blank 


\section{Preface}

No book is written in a vacuum. We would like to thank our colleagues for numerous discussions about many of the topics in this book. For many insights we are especially indebted to Claude Bernard, Poul Damgaard, Christof Gattringer, Maarten Golterman, Steve Gottlieb, Anna Hasenfratz, Peter Hasenfratz, Urs Heller, Jim Hetrick, Karl Jansen, Francesco Knechtli, Támas Kovács, Christian Lang, Martin Lüscher, Ferenc Niedermayer, Kostas Orginos, Stefan Schaefer, Yigal Shamir, Steve Sharpe, Rainer Sommer, Bob Sugar, Doug Toussaint, Peter Weisz, Hartmut Wittig, and Ulli Wolff. Any flaws are our own, however. Our research has been supported for many years by the U. S. National Science Foundation and Department of Energy.

Thomas DeGrand, Carleton DeTar 
This page is intentionally left blank 


\section{Contents}

Preface vii

1. Introduction 1

2. Continuum QCD and its phenomenology 5

2.1 The Lagrangian and QCD at short distance . . . . . . . 6

2.2 The nonrelativistic quark model . . . . . . . . . . 7

2.2.1 Explanations and puzzles . . . . . . . . . 7

2.2.2 Quark model interpolating operators . . . . . . . 9

2.2.3 Glueballs, hybrids, and exotics . . . . . . . . . 10

2.2.4 Flavor and glueball mixing example: the anomaly . . 11

2.2.5 Quark model hadron masses . . . . . . . . . . . 11

2.3 Heavy quark systems . . . . . . . . . . . . . . . . . 13

2.3 .1 Quarkonium . . . . . . . . . . . . . 13

2.3.2 Heavy quark symmetries . . . . . . . . . . . 14

2.4 Chiral symmetry and chiral symmetry breaking . . . . . 15

2.4.1 Chiral symmetry . . . . . . . . . . . . . 15

2.4 .2 Linear sigma model . . . . . . . . . . . . . . . 16

2.4.3 Nonlinear sigma model . . . . . . . . . . . . . 18

2.4.4 Nonlinear effective chiral Lagrangian for QCD . . . . 20

2.5 A technical aside: Ward identities . . . . . . . . . . 21

2.6 The axial anomaly and instantons . . . . . . . . . 24

2.6.1 Nonconservation of the flavor-singlet axial current . . 24

2.6.2 Witten-Veneziano formula . . . . . . . . . . 25

2.6.3 Suppression of the topological susceptibility . . . . 26

2.6 .4 QCD vacuum . . . . . . . . . . . 27 
2.7 The large $N_{c}$ limit $\ldots \ldots \ldots \ldots \ldots \ldots \ldots$

3. Path integration 33

3.1 Lattice Schwinger model . . . . . . . . . . . . . . . . 33

3.1 .1 Free fermions in one dimension . . . . . . . . . 33

3.1 .2 Species doubling . . . . . . . . . . . . 35

3.2 Hamiltonian with gauge fields . . . . . . . . . 36

3.3 Feynman path integral . . . . . . . . . . . . . 38

3.3 .1 Pure gauge theory . . . . . . . . . . . . 39

3.3.2 Generalization to $S U(3)$ pure gauge theory $\ldots \ldots .43$

3.3.3 Static quark potential . . . . . . . . . . . . 45

3.3.3.1 Polyakov loops and potential energy of point charges ................ 45

3.3.3.2 Wilson loops and point charge potential . . 46

3.4 Free fermions . . . . . . . . . . . . . . . 48

3.4.1 Grassmann calculus . . . . . . . . . . . . . . 48

3.4.2 Grassmann path integral for a two-level system . . . 50

3.4 .3 Fermion propagator . . . . . . . . . . . . 52

3.4.4 Path integral with particles and antiparticles . . . 54

3.4.5 Generalization to higher dimensions . . . . . . 56

3.5 The interacting theory . . . . . . . . . . . . 57

3.5.1 Functional integral representation for the lattice Schwinger model . . . . . . . . . . . 57

4. Renormalization and the renormalization group 61

4.1 Blocking transformations . . . . . . . . . . . 61

4.2 Renormalization group equations . . . . . . . . . . 71

4.2.1 Renormalization in scalar field theory . . . . . . . . 72

4.3 Renormalization group equations for the scalar field . . . 77

4.4 Effective field theories . . . . . . . . . . . . . 79

5. Yang-Mills theory on the lattice 87

5.1 Gauge invariance on the lattice . . . . . . . . . 87

5.2 Yang-Mills actions . . . . . . . . . . . . . 89

5.3 Gauge fixing $\ldots \ldots \ldots \ldots \ldots$

5.3 .1 Maximal tree gauge . . . . . . . . . . . . . . 90

5.3 .2 Landau and Coulomb gauge . . . . . . . . . . . 91

5.4 Strong coupling . . . . . . . . . . . . . . . . 92 
5.4.1 Wilson loop and confinement . . . . . . . . . 93

5.4 .2 Glueball mass . . . . . . . . . . . . . . 96

5.4 .3 Polyakov loop . . . . . . . . . . . . . . 98

6. Fermions on the lattice 101

6.1 Naive fermions . . . . . . . . . . . . . . . 101

6.2 Wilson-type fermions . . . . . . . . . . . . . 106

6.2 .1 Twisted-mass fermions . . . . . . . . . . . . 113

6.3 Staggered fermions . . . . . . . . . . . . . . 115

6.4 Lattice fermions with exact chiral symmetry . . . . . . . . 122

6.5 Exact chiral symmetry from five dimensions . . . . . . . 127

6.5.1 Five dimensions in the continuum . . . . . . . . 127

6.5.2 Five dimensions on the lattice . . . . . . . . . 130

6.6 Heavy quarks . . . . . . . . . . . . . . . . . 134

6.6.1 Heavy quark effective theory . . . . . . . . . . . 135

6.6 .2 Nonrelativistic QCD . . . . . . . . . . . . 137

6.6.3 Heavy, relativistic quarks . . . . . . . . . . . . 141

7. Numerical methods for bosons 143

7.1 Importance sampling . . . . . . . . . . . . . . . . 143

7.1.1 Monte Carlo methods . . . . . . . . . . . . . 144

7.1.1.1 Metropolis et al. method . . . . . . . . 145

7.1.1.2 Heat bath method . . . . . . . . . . . 145

7.1 .2 Molecular dynamics method . . . . . . . . . 146

7.1.3 Refreshed molecular dynamics . . . . . . . . . 147

7.1.4 Hybrid Monte Carlo . . . . . . . . . . . . . . . 148

7.1.5 Leapfrog algorithm and improvements . . . . . . . 148

7.2 Special methods for the Yang-Mills action . . . . . . . 150

7.2 .1 Heat bath . . . . . . . . . . . . 150

7.2 .2 Overrelaxed updates . . . . . . . . . . . 151

7.2 .3 Molecular dynamics . . . . . . . . . . . . . . 152

8. Numerical methods for fermions $\quad 155$

8.1 Taming the fermion determinant: the $\Phi$ algorithm $\ldots \ldots 155$

8.2 Taming the fermion determinant: the $\mathrm{R}$ algorithm $\ldots \ldots .159$

8.3 The fourth root approximation . . . . . . . . 160

8.4 An exact algorithm for the fourth root: rational hybrid Monte Carlo . . . . . . . . . . . . . . . . . . 162 
8.5 Refinements . . . . . . . . . . . . . . 163

8.5.1 Sexton-Weingarten scheme . . . . . . . . . 163

8.5 .2 Hasenbusch method . . . . . . . . . . . . . 163

8.5.3 Schwarz alternating method . . . . . . . . . 164

8.6 Special considerations for overlap fermions . . . . . . . . 165

8.7 Monte Carlo methods for fermions . . . . . . . . . . 170

8.7.1 Multiboson method . . . . . . . . . . . . 171

8.7 .2 Ratio of determinants . . . . . . . . . . . 171

8.8 Conjugate gradient and its relatives $\ldots \ldots \ldots \ldots$

8.8.1 Even-odd preconditioning . . . . . . . . . . 174

8.8.2 The conjugate gradient algorithm . . . . . . . 175

8.8 .3 Biconjugate gradient . . . . . . . . . . . . 178

8.8.4 Stabilized biconjugate gradient . . . . . . . . . . 179

8.8 .5 Shifted solvers . . . . . . . . . . . . . . . . 180

8.8.6 Computing fermion eigenmodes . . . . . . . . . 181

9. Data analysis for lattice simulations 183

9.1 Correlations in simulation time . . . . . . . . . . 184

9.2 Correlations among observables . . . . . . . . . . 186

9.2.1 Correlated least chi square . . . . . . . . . . 186

9.2.2 Truncating the correlation matrix . . . . . . . 187

9.2.3 Jackknife and bootstrap methods . . . . . . . . . 189

9.3 Fitting strategies . . . . . . . . . . . . . . 191

9.3.1 Fitting range . . . . . . . . . . . . . 191

9.3 .2 Signal to noise ratio . . . . . . . . . . . . . 192

9.3.3 Interpolating operator . . . . . . . . . . . . . 192

9.3.4 Asymmetric lattice . . . . . . . . . . . . . . . . 193

9.3.5 Bayesian methods . . . . . . . . . . . . . . . 194

10. Designing lattice actions 197

10.1 Motivation . . . . . . . . . . . . . . . . 197

10.2 Symanzik improvement . . . . . . . . . . . . . 199

10.2.1 Gauge action improvement . . . . . . . . . . 200

10.2.2 Fermion action improvement . . . . . . . . . . 201

10.2.3 Nonperturbative improvement . . . . . . . . . . . 202

10.3 Tadpole improvement . . . . . . . . . . . . . 204

10.4 Renormalization-group inspired improvement . . . . . . . 209

10.5 "Fat link" actions . . . . . . . . . . . . . . . . . 211 
11. Spectroscopy 215

11.1 Computing propagators and correlation functions . . . . . 215

11.2 Sewing propagators together . . . . . . . . . . . . . 219

11.2.1 Mesonic correlators - Wilson fermion formalism . . . 219

11.2.2 Baryonic correlators - Wilson fermion formalism . . 222

11.2.3 Mesonic correlators - staggered fermion formalism . 224

11.2.3.1 Flavor nonsinglet mesons . . . . . . . . . 225

11.2.3.2 Flavor singlet mesons . . . . . . . . . . 229

11.2.4 Baryonic correlators - staggered fermion formalism . 229

11.3 Glueballs . . . . . . . . . . . . . . . . 232

11.4 The string tension $\ldots \ldots \ldots \ldots \ldots$

12. Lattice perturbation theory 235

12.1 Motivation . . . . . . . . . . . . . 235

12.2 Technology . . . . . . . . . . . . . . 235

12.2.1 Free fermion and gluon propagators . . . . . . . 236

12.2 .2 Quark-gluon vertices . . . . . . . . . . . . 238

12.2.3 Fat links . . . . . . . . . . . . . . . . 239

12.2.4 Quark self energy and tadpole . . . . . . . . . 241

12.2.5 Vertex graph . . . . . . . . . . . . . . . . 242

12.2.6 Ultraviolet divergences . . . . . . . . . . . . . . . 243

12.2.7 Automation . . . . . . . . . . . . . . 243

12.3 The scale of the coupling constant $\ldots \ldots \ldots . \ldots 244$

13. Operators with anomalous dimension 249

13.1 Perturbative techniques for operator matching . . . . 250

13.2 Nonperturbative techniques for operator matching . . . . 253

13.2.1 Methods for approximations to conserved currents . 253

13.2.2 Regularization-independent scheme . . . . . . . 254

13.2.3 Schrödinger functional methods . . . . . . . . . 258

14. Chiral symmetry and lattice simulations 261

14.1 Minimal introduction to chiral perturbation theory . . . . . 261

14.2 Quenching, partial quenching, and unquenching . . . . . 265

14.2.1 The eta prime correlator . . . . . . . . . . 266

14.2.2 Chiral Lagrangian with partial or complete quenching 268

14.3 Chiral perturbation theory for staggered fermions . . . . 270

14.3.1 Staggered chiral perturbation theory . . . . . 270 
14.3.2 Rooted staggered chiral perturbation theory . . . . 273 14.4 Computing topological charge $\ldots \ldots \ldots \ldots 275$

15. Finite volume effects 279

15.1 Finite volume effects in chiral perturbation theory . . . . 279

15.2 The $\epsilon$-regime . . . . . . . . . . . . . . . . 282

15.2.1 Banks-Casher formula . . . . . . . . . . . 282

15.2.2 Chiral Lagrangian in the epsilon regime . . . . . . 283

15.2.3 Random matrix theory . . . . . . . . . . . . 284

15.2.4 Further applications . . . . . . . . . . 286

15.3 Finite volume, more generally . . . . . . . . . . . . 286

15.3.1 Single particle states . . . . . . . . . . . . 286

15.3.2 Two particle states . . . . . . . . . . . . 288

15.3.2.1 Scattering lengths from the two-particle ground state . . . . . . . . . . 289

15.3.2.2 Decay widths from level repulsion . . . . . . 290

15.4 Miscellaneous comments . . . . . . . . . . . . . 293

16. Testing the standard model with lattice calculations 295

16.1 Overview . . . . . . . . . . . . . . 295

16.2 Strong renormalization of weak operators . . . . . . 295

16.2.1 Effective Hamiltonian . . . . . . . . . . . . . . 295

16.2.2 An example: $c \rightarrow s \bar{d} u \ldots \ldots$. . . . . . . . . 297

16.2.3 Lattice $v s$ continuum renormalization of the effective Hamiltonian . . . . . . . . . . . . . . 300

16.2.4 Mixing with operators of higher and lower dimension 301

16.3 Lattice discrete symmetries . . . . . . . . . . . . . 303

16.4 Some simple examples . . . . . . . . . . . . . 306

16.4.1 Leptonic decay constants of mesons . . . . . . . . . 306

16.4.2 Leptonic decay constants in the heavy quark limit . . 307

16.4.3 Electromagnetic widths of vector mesons . . . . . . 308

16.4.4 Form factors . . . . . . . . . . . . . . . . . 308

16.4.5 Meson $B$ parameters . . . . . . . . . . . . 310

16.4.6 Other purely hadronic weak interactions . . . . . . 314

16.5 Evading a no-go theorem . . . . . . . . . . . 314

17. QCD at high temperature and density 317

17.1 Simulating high temperature . . . . . . . . . 317 
17.2 Introducing a chemical potential $\ldots \ldots \ldots . \ldots 318$

17.3 High quark mass limit and chiral limit . . . . . . . . 318

17.4 Locating and characterizing the phase transition . . . . 320

17.5 Simulating in a nearby ensemble . . . . . . . 320

17.6 Dimensional reduction and nonperturbative behavior . . . . 322

17.7 Miscellaneous observables . . . . . . . . . . . . . . 323

17.7.1 Quark number susceptibilities . . . . . . . . . 323

17.7.2 Quarkonium potential . . . . . . . . . . . 323

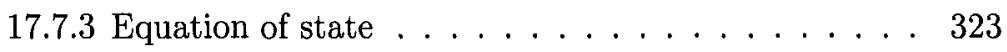

17.8 Nonzero density . . . . . . . . . . . . . . . . 325

17.9 Spectral functions and maximum entropy . . . . . . . 326

$\begin{array}{ll}\text { Bibliography } & 329\end{array}$

$\begin{array}{ll}\text { Index } & 341\end{array}$ 